TWO-DIMENSIONAL GAS FLOW IN AN ELECTRODE ASSEMBLY

Gordon W. Hamilton

Peter A. Willmann

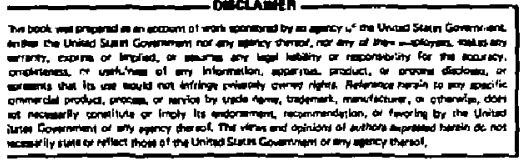




\section{TWO-DIMENSIONAL GAS FLOW IN AN ELECTRODE ASSEMBLY}

\section{ABSTRACT}

The interface between a source of positive or negative ions and a multichannel MEQALAC accelerator wil1 be the Low-Energy Beam Transport (LEBT) consisting of a lattice of quadrupole focusing electrodes transporting the beam while the gas pressure is reduced from the high-pressure ion source to the low-pressure accelerator. Gas emitted from the ion source will flow through the LEBT electrode lattice to a pumping volume. It is necessary to analyze the two-dimensional gas flow to ascertain the gas densities throughout the LEBT and to design the system so that only a small fraction of the ion beam is lost by gas collisions. The analysis uses the fact that the gas-flow rate is proportional to the density gradient if the mean free path of the low-pressure gas is greater than the inter-electrode spacing. Consequently the mathematics developed for conductivity of heat or electric current can be used.

The practical result of this analysis is to determine the maximum widtn of the LEBT so that the bean loss by gas collisions is tolerable. The maximum width is a function of beam density, gas efficiency, and electrode spacing. The beam current per unit length of module will be somewhat greater than 75 $\mathrm{mA} / \mathrm{cm}$. Electrode dimensions should be chosen to fit the characteristics of the ion source.

\section{INTRODUCTION}

A Low-Energy Beam Transport (LEBT) assembly is required to provide the interface between an ion source and a multi-channel rf accelerator, MEQALAC (Maschke, 1979). The LEBT is required to transport several thousand smal1 beams (about $6 \mathrm{~mA}, 20 \mathrm{keV}$ ) of positive or negative ions from the high-pressure ion source to the low-pressure accelerator. Beam transport is accomplished by a lattice of small, electrostatic, quadrupole electrodes that transport each small beam indiyidually by electrostatic strong focusing.

The LEBT and the gas pumping system must be designed so that the bear loss due to collisions with the background gas will not be excessive either in the low-energy LEBT or in the high-energy MEQALAC. He can tolerate about $10 \%$ beam loss in the LEBT but less than $1 \%$ beam loss in the MEQALAC after acceleration. Therefore the design requirements are as follows: 


$$
\int \mathrm{n} \sigma \mathrm{dT}<0.1 \text { in the LEBT, }
$$

and

$$
\int \text { n o d l }<0.01 \text { in the MEQALAC, }
$$

where the beam-loss cross-section, $\sigma$, refers to electron detachment of negative ions or electron capture by positive ions. The order of magnitude is 0 $=10^{-15} \mathrm{~cm}^{2}$ for both jositive and negative $H$ and $D$ ions in the LEBT energy range of 20 to $30 \mathrm{keV}$.

The gas density, $n$, is the sum of $n_{G}$, the density fixed by a pumping system outside the electrode assembly, and $\delta n$, the additional density caused by gas flow and density gradients within the electrode assembly. Since $n_{G}$ is measired outside the assembly and is spatially constant, (1) becomes the following for the LEBT:

$$
\int \mathrm{ndy}=\mathrm{n}_{\mathrm{G}} \mathrm{L}+\int_{0}^{!} \delta n d y<\frac{0.1}{\sigma} \approx 10^{14} \text { molecules } / \mathrm{cm}^{2} .
$$

The first term in Eq. (2) can be reduced to an arbitrarily low fraction by designing the external pumping system appropriately. However, the second term, $\int_{0}^{L}$ in $d y$, depends only upon the gas load and electrode dimensions affecting the gas flow through the electrode lattice. A gas-flow computation in two or three dimensions is required to evaluate this term.

TWO-DIMENSIONAL GAS-FLOW MODEL

The LEBT can be idealized as a two-dimensional slab, shown by Fig. 1, for purposes of gas-flow computations. Gas is injected into the LEBT from the ion source and is pumped out through the two sides. It is possible that the LEBT will consist of several thin slabs separated by pumping volumes or cryopanels. The length and width of the LEBT are the dimensions $L$ and $W$; the third dimension, $H$, may be considered infitite in comparison with $W$. A gas barrier between the LEBT and the MEQALAC accelerator will reduce the gas flow into the accelerator to a level that is negligible in respect to the LEBT but is not negligible in respect to the high-vacuum accelerator. 


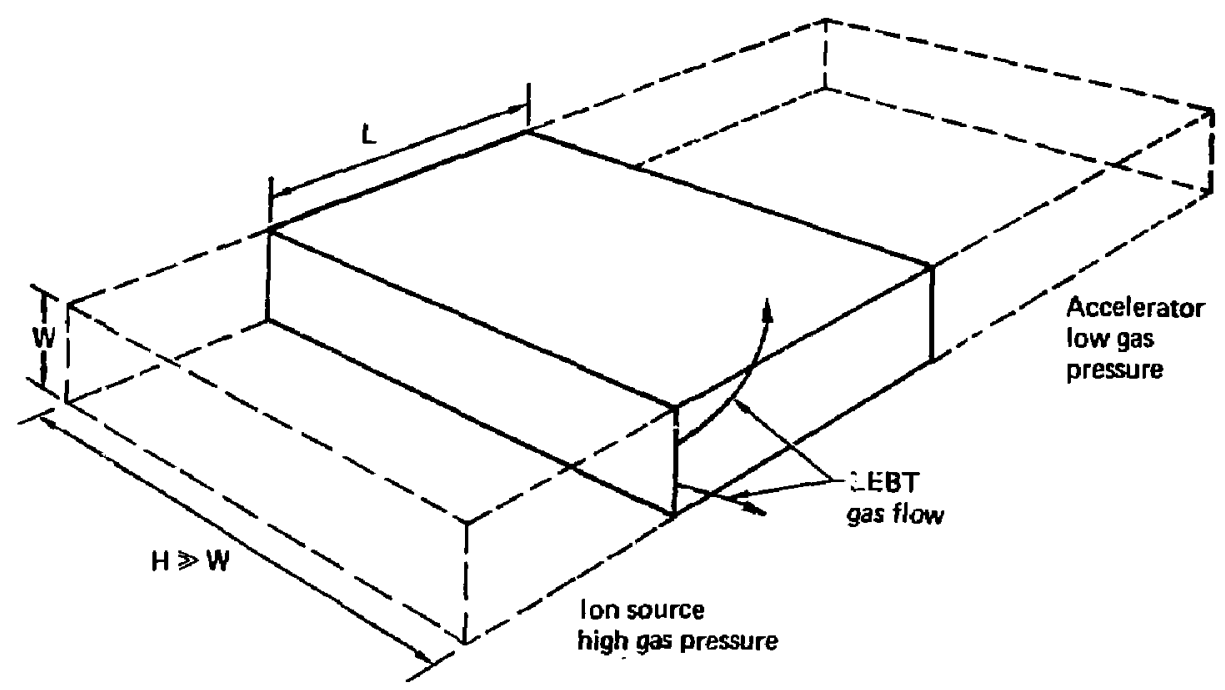

FIG. 1. Low Energy Beam Transport (LEBT) between a high pressure ion source and a low pressure accelerator. Gas from the ion source flows through the LEBT and is pumped at the top and bottom surfaces of the LEBT. If dimension $H$ is much larger than dimension $W$, the gas flow is two-dimensional. The $x-y$ coordinate axes are indicated.

The spacings between electrodes ( 4 to $10 \mathrm{~mm}$ ) are smaller than the mean free paths of the gas molecules for gas densities less than $10^{15}$ molecules/ $\mathrm{cm}^{3}$. Therefore the gas flow is molecular and is proportional to the density gradient. The gas flow equation is similar to the equations for flow of heat or electric current through a body according to its thermal or electric conductivity:

$$
\vec{F}=-\tilde{k} \cdot \nabla n \text { molecules } / \mathrm{cm}^{2}-\mathrm{s} \text {. }
$$

The conductivity, $\widetilde{k}$, is a tensor if the electrode lattice is not isotropic:

$$
\widetilde{k}=\left(\begin{array}{cc}
K_{x} & 0 \\
0 & x_{y}
\end{array}\right) .
$$

In steady state $\nabla \cdot \vec{F}=0$. If $\widetilde{K}$ is spatially constant and if subscripts are used to denote partial deriyatives of $n$, then equation (3) becomes the following: 


$$
k_{x} n_{x x}+k_{y} n_{y y}=0
$$

This can be converted to Laplace's equation by a change in variable:

$$
x=x^{\prime} k_{x} 1 / 2
$$

from which

$$
n_{x^{\prime} x^{\prime}}+n_{y^{\prime} y^{\prime}}=0
$$

This equation can be solved either numerically or analytically, subject to the boundary conditions. We will solve Eq. (7) analytically using the mathematics developed for heat flow problems after we evaluatie the gas conductivity, $\widetilde{k}$, and define the boundary conditions.

\section{EVALUATION OF $\tilde{\mathrm{K}}$}

The electrode lattice can be idealized as a series of grids. The spacings between grids are smaller than the external dimensions $W$ and $L$ but larger than the mean free path of the gas. Therefore, if the finite thickness of each grid is neglected, the conductivity per unit area of each grid is $3,640(\mathrm{~T} / \mathrm{M})^{1 / 2} \times$ grid transparancy in $\mathrm{cm} / \mathrm{s}$ (Van Atta, 1965). For $\mathrm{N}$ grids in series the conductivity per unit area is reduced by a factor of $N$.

The conductivity per unit volume $\left(\mathrm{cm}^{2} / \mathrm{s}\right)$ used in Eqs. (3) through (6) is therefore

$$
k_{x} \text { or } k_{y}=3,640(T / N)^{1 / 2} \text { (grid transparency) - (spacing between grids), }
$$

where the grid transparency $\left(G_{x}\right.$ or $G_{y}$ ) and grid spacing $\left(S_{x}\right.$ or $\left.S_{y}\right)$ in the $x$ direction may be different from the transparency and spacing in the $y$ direction. Equation (8) may be modified to include the finite thickness of each grid after the electrode details are better known.

Equation (8) is based on the assumption that the gas molecules are moving in random directions. This assumption may not be valid for the case of gas flow through a series of aligned apertures, such as the series of apertures defining the beam channels of the LEBT. It is usual in such cases to increase the conductivity by an estimated correction factor to take into account the 
fraction of molecules streaming through aligned apertures : thout collisions. However, the correction to $K$ because of aligned apertures will not be very important for three reasons: not $K_{x}$.

1. In the LEBT electrode lattice the aligned apertures affect on ly $K_{y}$,

2. It will turn out that $K_{y}$ cance 15 from the equations when the integra] $\int \delta n d y$ is computed. This will be discussed under Scaling Laws. The only importance of $K_{y}$ will be in determining the required length, $L$, of the LEBT. To minimize the required length, $L$, one must also minimize $k_{y}$.

3. The two corrections for finite length of electrodes and for airigned apertures wi]l tend to cancel each other. Both corrections should be applied as a final step in the design after the electrode details are better known.

\section{BOUNOARY CONDITIONS}

The boundary conditions stated in the description of the gas flow model can be converted to the notation of Eq. (7). In the $x^{\prime}-y^{\prime}$ coordinate system, we will define the dimensions a and $b$ :

$$
2 a / w=x \cdot / x=k_{x}-1 / 2
$$

and

$$
b / L=y^{\prime} / y=k_{y}-1 / 2 \text {. }
$$

The dimensions and the boundary conditions are indicated in Fig. 2 and are specified below.

1. At the ion source $y=y^{\prime}=0$ and the gas flow, $F_{0}$, is determined by the average beam current density, $J$, and the ion source gas efficiency, $E$. Let $A$ equal the average number of atoms in a beam ion. $\left(A=1\right.$ for a $D^{-}$ beam; $A=1.2$ for a mixture of $D^{+}, D_{2}^{+}$, and $D_{3}^{+}$) Then,

$$
\begin{aligned}
F_{0} & =A J /(2 E q) \text { molecu }] \text { es } / \mathrm{cm}^{2}-s \\
& =-K_{y^{n}} n_{y}=-K_{y}^{1 / 2} n_{y^{\prime}},
\end{aligned}
$$

where we have used Eqs. (3) and (6). The boundary condition is 


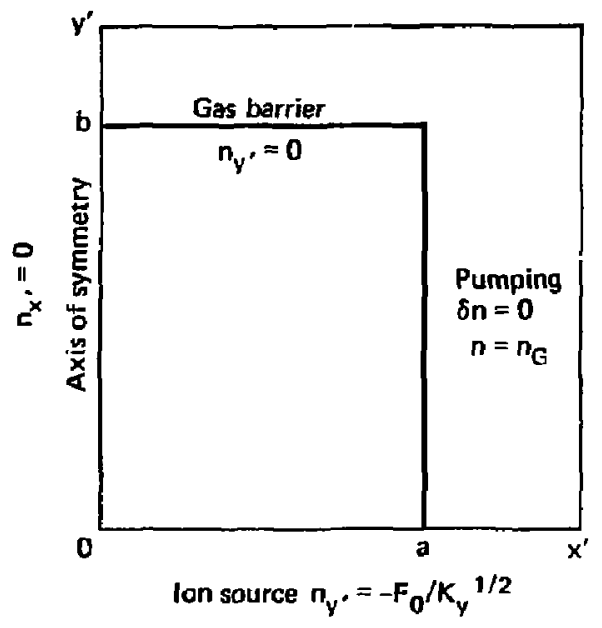

FIG. 2. Boundary conditions in $x^{\prime}-y^{\prime}$ coordinate system. Subscripts of $n$ denote partial derivatives:

a. at the ion source $y^{\prime}=0$ and $n_{y^{\prime}}=-F_{0} / \mathrm{Kb}^{\prime} / 2$;

b. at the gas barrier $y^{\prime}=b$ and $n_{y^{\prime}}=0$;

c. at the axis of symetry $x^{\prime}=0$ and $n_{x^{\prime}}=0$;

d. at the boundary of the gas pumping region, $x^{\prime}=a$ and $n=n_{G}$.

$n_{y^{\prime}}=-F_{0} k_{y}^{1 / 2}$ at $y^{\prime}=0$.

2. At the gas barrier $y=L, y^{\prime}=b$, and there is no gas flow, i.e.,

$n_{y^{\prime}}=0$ at $y^{\prime}=b$.

3. At the axis of symetry $x=x^{\prime}=0$, and there is no gas flow or density gradient in the $x^{\prime}$ direction, i.e.,

$$
n_{x^{\prime}}=0 \text { at } x^{\prime}=0 \text {. }
$$

4. At the boundary of the gas pumping region, $x=W / 2, x^{\prime}=a$, and $n=$ $\pi_{G}$, i.e.,

$$
n=n_{G} \text { at } x^{\prime}=\mathbf{a} \text {. }
$$


We can solve Eq. (7) subject to tre boundary conditions by using the methods deveioped for heat transfer problems (Carslaw and Jaeger, 1959, Ch. v). A solution that satisfied Eqs. (7), (12), and (13) is

$$
n\left(x^{\prime}, y^{\prime}\right)=n_{G}+\sum_{n=1}^{\infty} a_{n} \cos a_{n} x^{\prime} \cosh a_{n}\left(b-y^{\prime}\right),
$$

where the coefficients $a_{n}$ and $a_{n}$ are to be derived from (10) and (14).

The boundary condition (14) requires that $\cos a_{n} a=0$, and consequently,

$$
a_{n}=(n-1 / 2) \pi / a .
$$

To evaluate the coefficients $a_{n}$, fjrst combine equations (11) and (15), where $y^{\prime}=0$ :

$$
n_{y^{\prime}}=-F_{0^{\prime}} / k_{y}^{1 / 2}=-\sum_{n=1}^{\infty} a_{n} \cos a_{n} x^{\prime} \sinh a_{n} b a_{n} .
$$

Now multiply by $\cos a_{\mathrm{m}^{\prime}}$ and integrate term by term. The left side is

$$
\begin{aligned}
L S & =\int_{0}^{a}-\left(F_{0} / k_{y}^{1 / 2}\right) \cos a_{m} x^{\prime} d x^{\prime}, \\
& =-\left(F_{0} / a_{m} k_{y}^{1 / 2}\right) \sin [(m-1 / 2) \pi], \\
& =+\left(F_{0} a_{m} k_{y}^{1 / 2}\right)(-1)^{m} .
\end{aligned}
$$

If one uses Eq. (16) and $u=\pi x / a$, the right side of (17) can be integrated as follows: 
$R S=-\sum_{n=1}^{\infty} a_{n} a_{n} \sinh a_{n}^{b} \int_{u=0}^{\pi} \cos (n-1 / 2) u \cos (m-1 / 2) u d u(a / n)$

If one uses a trigonometric identity, the integral becoires

$$
\begin{aligned}
\int_{u=0}^{\pi} \frac{1}{2}[\cos (n+m-1) u+\cos (n-m) u] d u & \\
& =\frac{1}{2}\left[\frac{\sin (n+m-1) u}{(n+m-1)}+\frac{\sin (n-m) u}{(n-m)}\right]_{u=0}^{\pi} .
\end{aligned}
$$

If $n$ is not equal to $m$, the result in $(20)$ is always zero. The coefficient $(n+m-1)$ is always greater than zero, because the mininum values of $n$ and $m$ is one. The only ron-zero tem in the integration is derived from the second term of the integrand in (20), for which $(n-m) u=0$. The result of the integration is $\pi / 2$, and Eq. (19) becomes

$$
\mathrm{RS}=-1 / 2 \mathrm{a} a_{m} a_{m} \sinh a_{m} b
$$

Now set the LS (18) equal to RS (21); change the subscript m to $n$; and solve for the coefficient $a_{n}$ :

$$
a_{n}=\frac{-2 F_{0}(-1)^{n}}{k_{y}^{1 / 2} a_{n}^{2} a} \sinh a_{n}^{b}
$$

The solution (15) now becones

$$
n\left(x^{\prime}, y^{\prime}\right)=n_{G}-\frac{2 F_{0}}{K_{y}^{1 / 2} a} \sum_{n=1}^{\infty} \frac{(-1)^{n} \cos a_{n} x^{\prime} \cosh a_{n}\left(b-y^{\prime}\right)}{a_{n}^{2} \sinh a_{n} b} .
$$

With the notation converted back to the $x-y$ system, the solution is 


$$
n(x, y)=n_{G}+n=n_{G}-\frac{F_{0} W}{4 \pi^{2}\left(K_{x} K_{y}\right)^{1 / 2}} \sum_{n=1}^{\infty} A_{n},
$$

where

$$
A_{n}=(-1)^{n} \frac{\cos [2 \pi(n-1 / 2) x / w] \cosh [-(n-1 / 2) R(1-y / L)]}{(n-1 / 2)^{2} \sinh [\pi(n-1 / 2) R]} \text {, }
$$

and where $R$ is the aspect ratio $L / W$ corrected for the anisotropy of $K$ :

$$
R_{i}=(L / W)\left(K_{x} / K_{y}\right)^{1 / 2}
$$

\section{COMPUTATIONS}

Equations (16) and (23) have been programed into GASOUT to compute $\delta n\left(x, y^{\prime}\right.$ and $\int_{0}^{L} \delta n(x, y) d y$, and to display the results in tables and grapts. For each case the required input data are $F_{0}, K_{x}, K_{y}$, and $L . A$ prelimirary computation by hand is required for $F_{0}, K_{x}$, and $k_{y}$, by using Eqs. (8) and (10) rith specified values of grid transparency $G_{x}$ and $G_{y}$, grid spacing $S_{x}$ and $S_{y}$, beam current density $J$, and gâs efficiency $E$. The width, $W$, is varied frota $2 \mathrm{~cm}$ to $20 \mathrm{~cm}$ in order to find the maximum a:lowable width for which $\int \delta n(x, y)$ ay does not exceed $10^{14}$ molecules $/ \mathrm{cm}^{2}$.

We have verified the correctness of the solution by making sure that the computer output $\delta n(x, y)$ satisfies the four boundary conditions and by ascertaining that $\delta n(0,0)$ is proportional to the width, $W$, and agrees in order of magnitude with a one-dimenjional approximation. The program GASOUT inc?udis a test for convergence of the infinite series (25).

The computer cannot handle the cosh or sinh when the argument in (23) becomes 1 arge. This proislem was overcome by a very good approximation:

$$
\cosh u \text { or } \sinh u=e^{u} / 2 \text { when } u>6 \text {. }
$$

Consequently, for large arguments, the ratio $\cosh a_{n}\left(b-y^{\prime}\right) / \sinh a_{n} b$ can be replaced with $\exp \left(-a_{n} y^{\prime}\right)$. This replacement speeded up the computation of (23) and avoided computer overflow. 


\section{INPUT CONDITIONS}

Three sets of input conditions have been used as examples for GASOUT computations. The firs: two conditions are for a low-density example (suggested by Joel Fink), in which we used two assumptions for the electrode spacings, thereby bracketing the actual conditions. The third input condition was based upon a high density layout (produced by A. W. Maschike) witl, 6-mA Dbeams spaced 4-m apart. The three sets of input cuititions are specified in Table 1.

\section{RESULTS OF GASOUT COMPUTATIONS}

The results of GASOUT computations consist of tabulatians and contour plots showing absolute values of $\delta n(x, y)$ for each set of input conditions. The integrated thickness $\int \delta n(x, y)$ dy is computed for ten values of $x$ and displayed in anc able and graph. Figure 3 shows some contours of gas densities for suecified conditions and Figure 4 shows the integrated thicknesses as functions of the width, $W$, for 10 values of $x / W$.

The most practical result of these computations is the maximum allowable value of $W$ for which the integrated gas thickness does not exceed $10^{14}$ molecules $/ \mathrm{cm}^{2}$. Therefore we specify 10 values of $W$ in the range from 2 to $20 \mathrm{~cm}$ for each input condition and obtain 10 sets of results. By interpolating, we find the maximum allowable $W$, which is also indicated in Table 1 . For the low-density example the maximum $w$ is somewhere between 7 and $14 \mathrm{~cm}$, depending upon the electrode spacing. For the high-density example, $W$ must not be greater than $2 \mathrm{~cm}$. Our layouts of the LEBT and ion source must $f$ it this constraint.

Larger assemblies are possible if they are built in layers of thickness not larger than $W$ with cryopanels or other gas pumping between layers. This requirement is compatible with modules transporting about two amperes of $\mathrm{D}^{-}$ or about six amperes of $D^{+}$. Any number of modules can be operated in parallel if adequate pumping is provided between modules.

\section{SCALING LAWS}

In order to optimize the design, it is useful to understand how the gas density and thickness scale with input conditions. Equation (24) indicates 
TABLE 1. Input conditions and results for three examples of GASOUT computations in Low Energy Beam Transport (LEBT).

\begin{tabular}{|c|c|}
\hline $\begin{array}{l}\text { Low density } \\
\text { (Fink) }\end{array}$ & $\begin{array}{l}\text { High density } \\
\text { (Maschke) }\end{array}$ \\
\hline Examp ie & \\
\hline
\end{tabular}

Input conditions

$\left.J(\mathrm{~mA} / \mathrm{cm})^{2}\right)$

E (gas efficiency)

A (atoms/ion)

$\mathrm{F}_{\mathrm{O}}=\mathrm{AJ} / 2 \mathrm{Eq}$ (molecules $/ \mathrm{cm}^{2}$-sec)

4.1

0.1

4.1

37.5

1

0.1

$12.8 \mathrm{El6}$

1

$12.8 \mathrm{E} 16$

0.1

1

Grid transparencies for gas flow computations
$G_{x}$
0.5
0.5
0.30
0.5
0.5
0.35

Assumed spacings between grids, cm

$$
S_{x}
$$

2

0.4

1

4

1.2

Gas conductivities, $\mathrm{cm}^{2} / \mathrm{s}$

$$
\begin{aligned}
& K_{x}=3,640(T / M)^{1 / 2} G_{x} S_{x} \\
& K_{y} \\
& T / M
\end{aligned}
$$

0.78 E4
1.56
72

3.12 E4

0.363 E4

6.24

72

1.26

$L$, length of LEBT, cm

18

18

18

Results of GASOUT computations for

$W=2$ to $20 \mathrm{~cm}$ (interpolated):

1. Maximum allowable W for

which $\int \mathrm{d}$ dy $<10^{14}$

$\mathrm{cm}^{-2}$. ( $\left.\mathrm{cm}\right)$

7

14

$<2 \mathrm{~cm}$

2. Maximum density $\delta$ n at

$x=0, y=0$. $\left(\mathrm{cm}^{-3}\right)$

$3.0 \mathrm{E} 12$

$1.5 \mathrm{E} 13$

$10 \mathrm{E} 13$

3. Maximum JW ( $\mathrm{mA} / \mathrm{cm})$

29

57

75

Note: Because of the present uncertainty about the grid spacing, we ran two examples of the low-density case to bracket the real condition. In the highdensity case we use $S_{x}=0.4 \mathrm{~cm}$, the spacing between channels. 


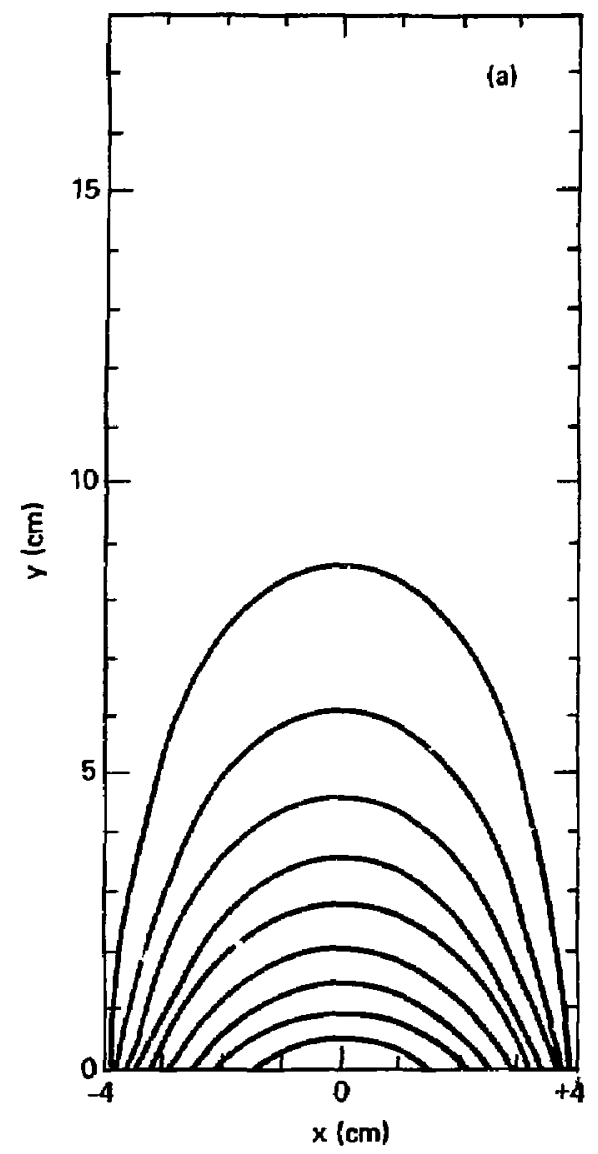

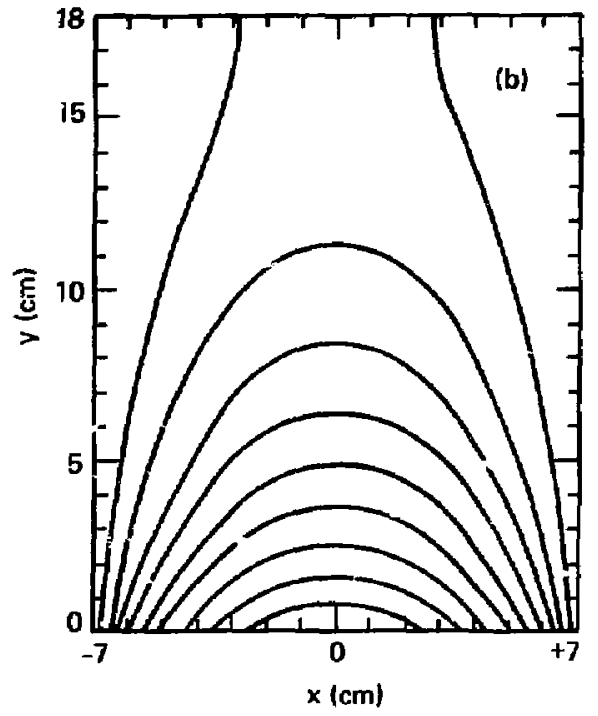

FIG. 3. Two samples of gas density contours. The contour interval is $8 \mathrm{n}(0,0) / 10$. The input conditions are listed in the second column of Table 1: (a) $W=8 \mathrm{~cm}$; (b) $W=14$ $\mathrm{cm}$.

that the maximum value of $\delta \mathrm{n}$ is proportional to $\mathrm{F}_{0} \mathrm{H}\left(\mathrm{K}_{x} \mathrm{~K}_{y}\right)^{1 / 2}$, if the aspect ratio, $R$, is large enough to avoid end effects.

Figure 3 indicates that the gas thickness $\int \delta$ in $d y$ is proportional to $W^{2}$ if the aspect ratio $R$ is large enough to avoid end effects. This is bacause the scale lengths of the density gradients in both the $x$ and $y$ directions are proportional to $\mathrm{W}$ :

$$
\int \delta n d y=\delta n w=F_{0} w^{2} /\left(K_{x} K_{y}\right)^{1 / 2} .
$$




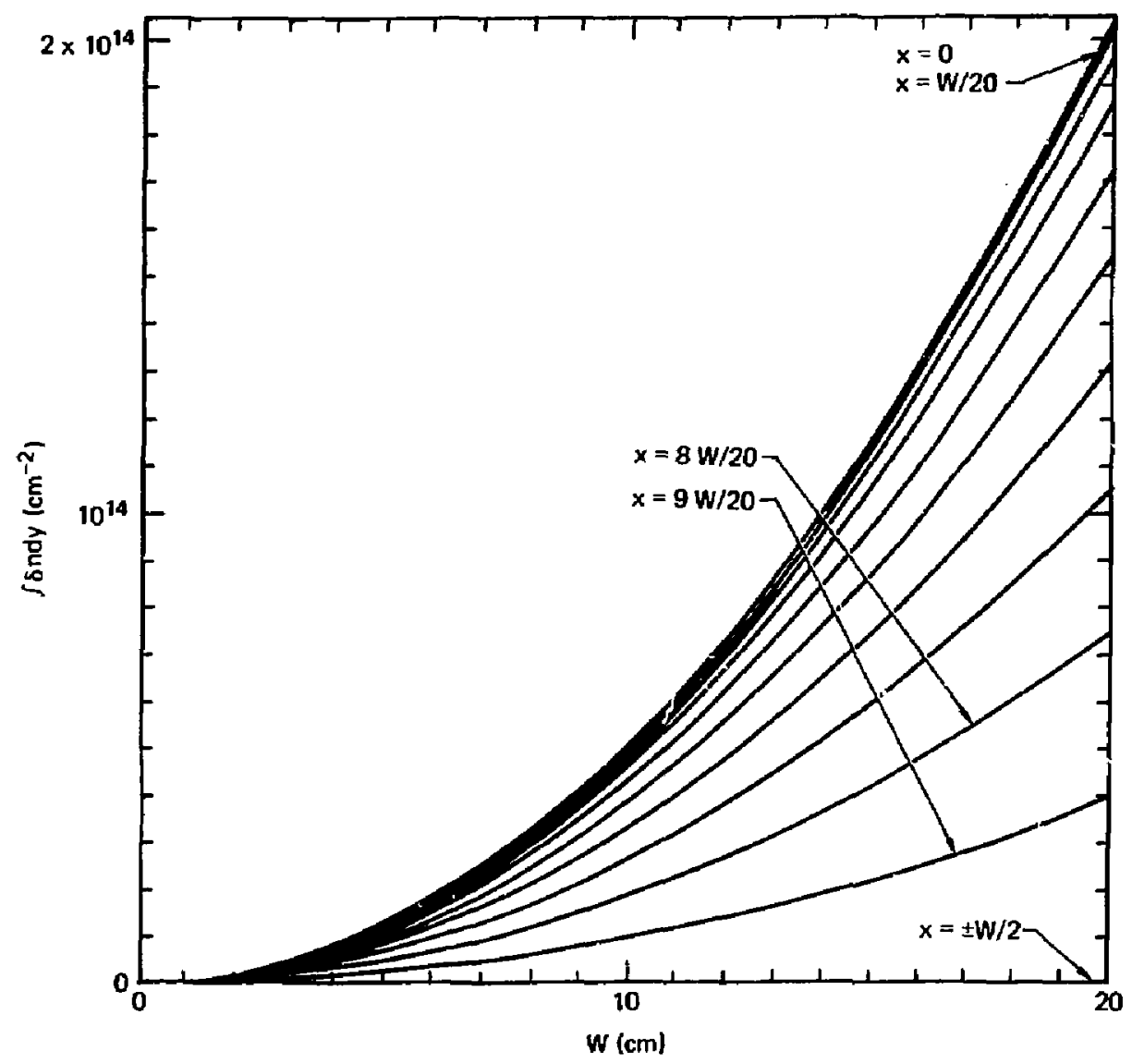

FIG. 4. Integrated gas thickness $\int \delta n$ dy as a function of the width, $W$, for ten values of $x / W$ varying from 0 to 0.5 . The input conditions are listed in the second columin of Table 1. For the maximum allowable gas thickness of $10^{14}$ molecules $/ \mathrm{cm}^{2}$, the maximum al lowable value of $W$ is $14 \mathrm{~cm}$.

The gas load, $F_{0}$, is proportional to the current density, J. We will probably want to maximize the current per unit length of the LEBT module, since the cryopanels between modules may occupy more space than the module itse If.

$$
\text { Current/ length }=J_{w} \frac{\left(K_{x} K_{y}\right)^{1 / 2}}{W} \int \delta n d y .
$$


The two constant factors are $\int \delta n d y \leq 10^{14}$ and current per channel $=$ $\mathrm{J} S_{x}^{2}=6 \mathrm{~mA}$. We can now ascertain how $J W$ depends upon $S_{x}$. We will assume that $S_{y}$ is proportional to $S_{x}$ but that the transparencies $G_{x}$ and $G_{y}$ are independent of $S_{x}$. From (27),

$$
\begin{aligned}
& 10^{14}-J H^{2} / S_{x}=\frac{(6 m A)}{S_{x}^{2}} \frac{W^{2}}{S_{x}}, \\
& W=S_{x}^{3 / 2},
\end{aligned}
$$

and from (28),

$$
J W=\frac{S_{x}}{W} \propto S_{x}^{-1 / 2}
$$

The integrated gas thickness may be expressed as the product of the maximum density and the scale length, SL, of the density gradient:

$$
\int \delta n d y=S L \delta n(0,0),
$$

where SL is proportional to $W$, corrected for the an isotropy of $K$,

$$
S L-W\left(K_{y} / K_{x}\right)^{1 / 2}
$$

Therefore, combining (27), (31), and (32), we find

$$
\int \delta n d y=F_{0} w^{2} / K_{x}
$$

which is independent of $K_{\mathbf{y}^{*}}$.

These scaling laws have been verified by GASOUT computations of $\delta \mathrm{n}(0,0)$ and $\int \delta n d y$. One of the conclusions is that $k_{y}$ does not affect $f_{\delta n} d y$. In the electrode design, $K_{x}$ should be as large as possible to increase the allowable width, $W$, but $K_{y}$ should be as small as possible to reduce the required length, L. However, the electrode dimensions should be selested to fit the characteristics of the ion source. 
If the LEBT consists of several layers of beam channels of width $W$ separated by cryopanels of width $C$, the overall bean current density will be

$$
\langle\mathrm{J}\rangle=J W /(W+C)=J /(1+C / W) .
$$

This can be converted to a function of the spacing $S_{x}$ by using Eq. $(30)$, scaling from the result sinown by the third column of Table I:

$$
J=6 m A / s_{x}^{2} ; \quad W=7.91 s_{x}^{3 / 2}
$$

Therefore,

$$
\langle\mathrm{J}\rangle=6 \mathrm{~mA} /\left(\mathrm{s}_{\mathrm{x}}^{2}+\mathrm{CS}_{\mathrm{x}}^{1 / 2} / 7.91\right)
$$

This result shows that the overall current density is improved with small dimensions even if $W<C$, $i . e$. , even if most of the area is occupied by cryopanels. For example, if the thickness of the cryopanels including baffles if $5 \mathrm{~cm},\langle\mathrm{~J}\rangle=10.7 \mathrm{~mA} / \mathrm{cm}^{2}$ if $\mathrm{s}_{x}=0.4 \mathrm{~cm}$. If it is possible to miniaturize the electrodes so that $S_{x}=0.1 \mathrm{~cm}$, then $\langle J\rangle$ is increased to $28.6 \mathrm{~mA} / \mathrm{cm}^{2}$. This compares favorably with the overall bean density of dc accelerators if we include the space required for high voltage insulation and gas pumping. 


\section{ADDENDUM}

\section{Intraduction}

The assumption of uniform gas density, $n_{G}$, in the pumping volume (which was made in the body of the paper) may not be realistic. This Addendum will treat the case in which the gas density in the pumping line is not uniform. A realistic layout showing cryopanels sandwiched between layers of the LEBT is shown by Figure Al, One of the facts obvious from this layout is that the pumping volume has a small conductance for gas flow in the $y$ direction in comparison to the pumping speed of the cryopaneis. Consequently the gas density at the interface between the LEBT and the cryopanels will be a function of the gas flow, $F_{X}$, out of the LEBT and the pumping speed, $S$, of the cryopanels; i.e., at $x=w / 2$,

$$
n(x, y)=F_{x} / S
$$

Since the gas flow, $F_{x}$, will be a function of $y$, the gas density will also te a function of $y$. This is contrary to the previous assumption of uniform gas density in the pumping vilume. We will now derjue a solution to the gas flow equation subject to this revised and more accurate boundary condition. As before, we can follow the mathematics of Cars law and Jaeger (1959, Ch. V) to obtain an analytical solution consistent with the revised boundary condition.

\section{Revised Boundary Condition}

The gas fiow out of the LEBT is

$$
F_{x}=-K_{x} n_{x}=S n \text { at } x=W / 2
$$

As before we are using subscripts to denote partial derivatives of $n$. After conversion to the $x^{\prime}-y^{\prime}$ system, this boundary condition becomes the following:

$$
-k_{x}^{1 / 2} n_{x},=S n \text { at } x=a
$$




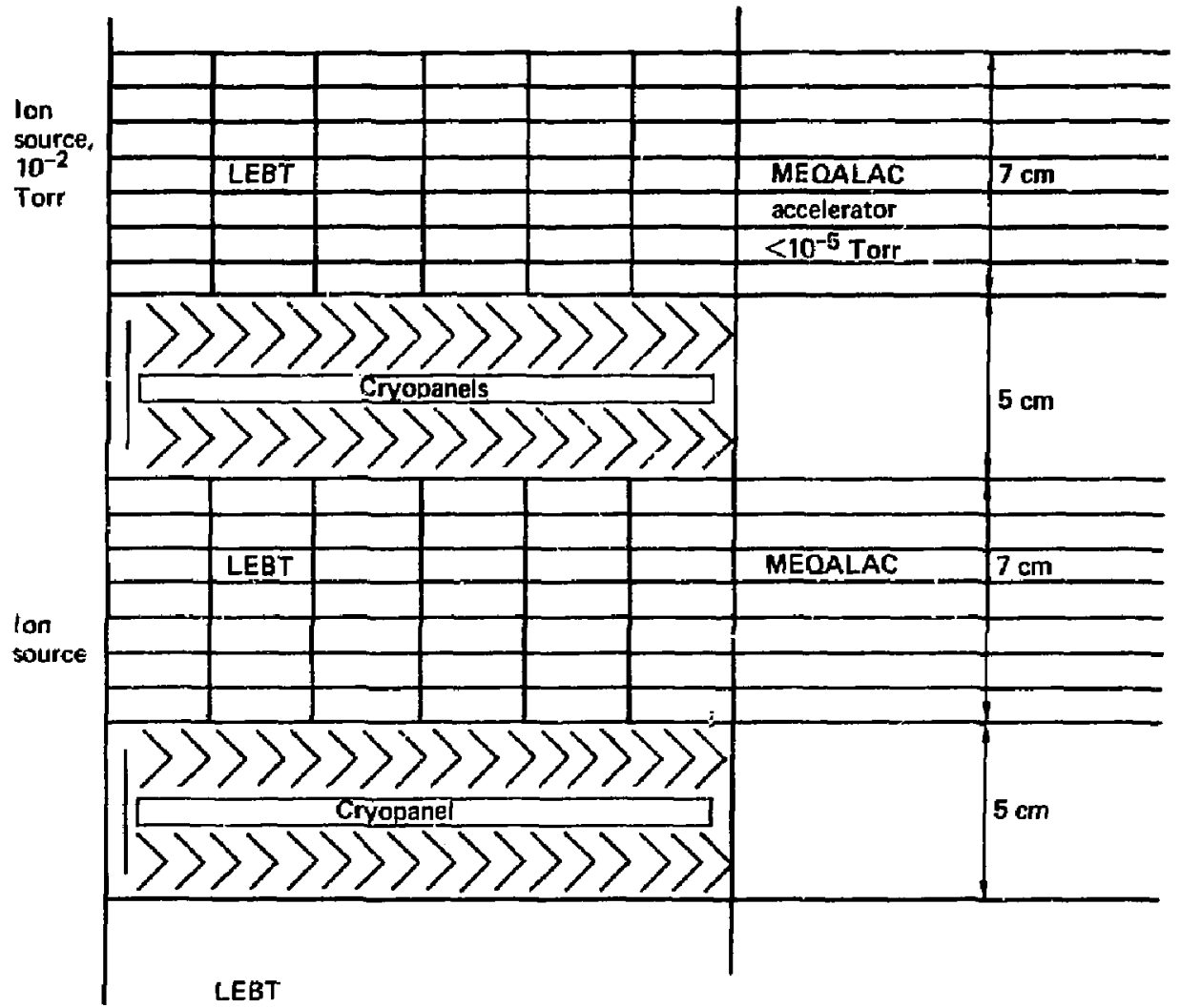

FIG. Al. Low Energy Beam Transport (LEBT) sandwiched between cryopanels. The system must reduce the gas pressure from about $10^{-2}$ Tarr at the ion source to less than $10^{-5}$ Torr in the accelerator. The pumping speed of the baffled cryopanels is 7 liter/s $-\mathrm{cm}^{-2}$.

or

$$
n_{x^{\prime}}+h n=0 \text { at } x^{\prime}=a \text {. }
$$

[Replaces (14)]

Here we have defined $h=S K_{x}^{-1 / 2}$ to conform with the notation of Cars law and Jaeger (1959, p. 19) for their radiation boundary condition. 


\section{Evaluation of Coefficients an}

We must use the revised boundary condition (A3) to evaluate the coefficients $a_{n}$ and $a_{n}$ in the analytical solution (15). By combining these two equations find a transcendental equation $a_{n}$ :

$$
a_{n} \tan a_{n} a^{a}=h
$$

When $h$ approaches infinity, Eq. (A4) becomes identical to the version given earlier in this paper as Eq. (16).

This equation must be solved iteratively for the $n$th positive root, ${ }^{n} n$. The roots of this equation are the intersections of the two functions $\tan a_{n} a$ and $h / a_{n}$, which are shown by Fig. A2. From this figure we know that each root is in the following interval:

$$
(n-1) \pi / a<a_{n}<(n-1 / 2) \pi / a \text {. }
$$

Knowing that the root is in this interval, we found the ru." : Subroutine ZEROIN, which is in the LLL computer library, MATHLIB. :towever, this subroutine does not work for large values of $n$, when $a_{n}$ is near the lower limit of this interval. We overcame this problem by a small-angle approximation:

$$
\tan \left(a_{n^{a}}\right)=a_{n} a-(n-1)_{\pi} \text { if } a_{n^{a}}-(n-1)_{\pi}<0.02
$$

By this approximation, (A4) becomes a quadratic equation which can be solved for the unknown $a_{n}$.

\section{Evaluation of Coefficients a}

Now we must find the coefficients $a_{n}$ by combining (11) and (15), multiplying each term by $\cos \alpha_{\pi} x$, and integrating. The procedure is the same as it was before, except thax (16) is replaced by (A4) and, therefore, $a^{a}{ }^{a}$ is not a integral multiple of $\pi / 2$. The left side of the integration, LS, (18) is not changed. 


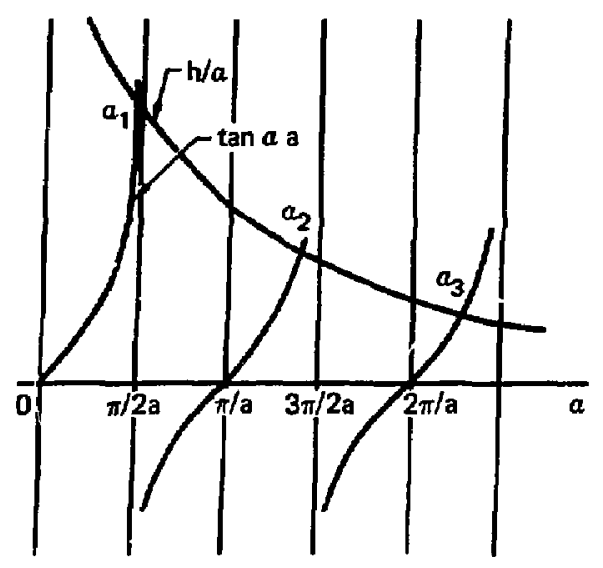

FIG. A2. The functions $\tan a_{n} a$ and $h / a_{n}$. The intersections of these two functions define the roots of equation (A4). Each intersection is somewhere within the interval shown by equation (AD).

To evaluate the right side of the integration, RS, [simi iar to (19)], we will refer to Carslaw and Jaeger (1959, p. 116) to prove the following:

$$
\int_{0}^{a} \cos a_{n} 5^{\prime} \cos a_{m} x^{\prime} d x^{\prime}=0
$$

if $n \neq m$ and if the coefficient $a_{n}$ is given by (A4). Consequent $1 y$, the only non-zero term in the sumnation (19) is

$$
R S=-a_{n} a_{n} \sinh a_{n}^{b} \int_{0}^{a} \cos ^{2} a_{n^{\prime}} x^{\prime} d x^{\prime}
$$

$$
\begin{aligned}
& =-a_{n} a \sinh a_{n} b\left[\sin a_{n} a \cos \alpha_{n} a+a_{n} a / 2\right] \\
& =-a_{n} a \sinh \alpha_{n} b\left[h a_{n} /\left(h^{2}+a_{n}^{2}\right)+a_{n} a / 2\right] .
\end{aligned}
$$

Here we have used (A4) to evaluate the sine and cosine. Now we find $a_{n}$ by setting the LS (18) equal to the RS (AB). The solution (15) now becomes the following: 


$$
n\left(x^{\prime}, y^{\prime}\right)=\frac{-F_{0}}{k_{y}^{1 / 2}} \sum_{n=1}^{\infty} \frac{(-1)^{n} \cos a_{n} x^{\prime} \cosh a_{n}\left(b-y^{\prime}\right)}{\left[\frac{h}{\left(h^{2}+a_{n}^{2}\right)}+\frac{a}{2}\right] a_{n}^{2} \sinh a_{n}^{b}} .
$$

Comparing (A9) with the previous solution (23), one sees the only change is replacement of a/2 with the expression in square brackets. Again the two versians become identical when $h$ approaches infinity.

\section{Computational Results}

Figure A3 shows contours of constant density computed for the two types of boundary conditions. GASOUT 1 has computed the gas densities assuming a uniform density, $n_{G}$, in the pumping volume while GASOUT 2 has computed the densities assuming cryopanels adjacent to the LEBT. The pumping speed, 5 , of baffled cryopanels is about 711 ter $/ \mathrm{s}-\mathrm{cm}^{2}$, or $7,000 \mathrm{~cm} / \mathrm{s}$. Other input conditions for these two examples are the same as in thie third colvimn of Table 1.

In comparing these two results, it is important to realize that the total gas density, $n(x, y)$, consists of two terms under the assumptions of GASOUT 1 but only one term under the assumptions of GASOUT 2;

$$
n(x, y)=n_{G}+\delta n(x, y) \text { for GASOUT } 1 \text {, }
$$

where the first term is computed by a separate calculation and where only $\delta n(x, y)$ is computed by GASOUT 1 . On the other hand, the total density, $n(x, y)$, is computed by GASOUT 2. This explains the difference between the contour intervals specified in the caption of Fig. A3.

The density contours of Fig. A3(a) clearly show that there is no density gradient along the boundaries of the pumping volume. On the other hand, the contours indicate that there is a density gradient along this boundary for GASOUT 2, Fig. A3(b). This tends to support the correctness of the two computations.

Figure A4 shows the indicated gas thicknesses computed by GASOUT 1 and GASOUT 2 for dimensions $W$ in the range up to $14 \mathrm{~cm}$. The relative correction is almost $100 \%$ for small values of $W$ but becomes less important for large $W$. Both computations indicate that $W$ must be less than about $2 \mathrm{~cm}$ for a yas thickness less than $2 \times 10^{14} \mathrm{~cm}^{-2}$. 

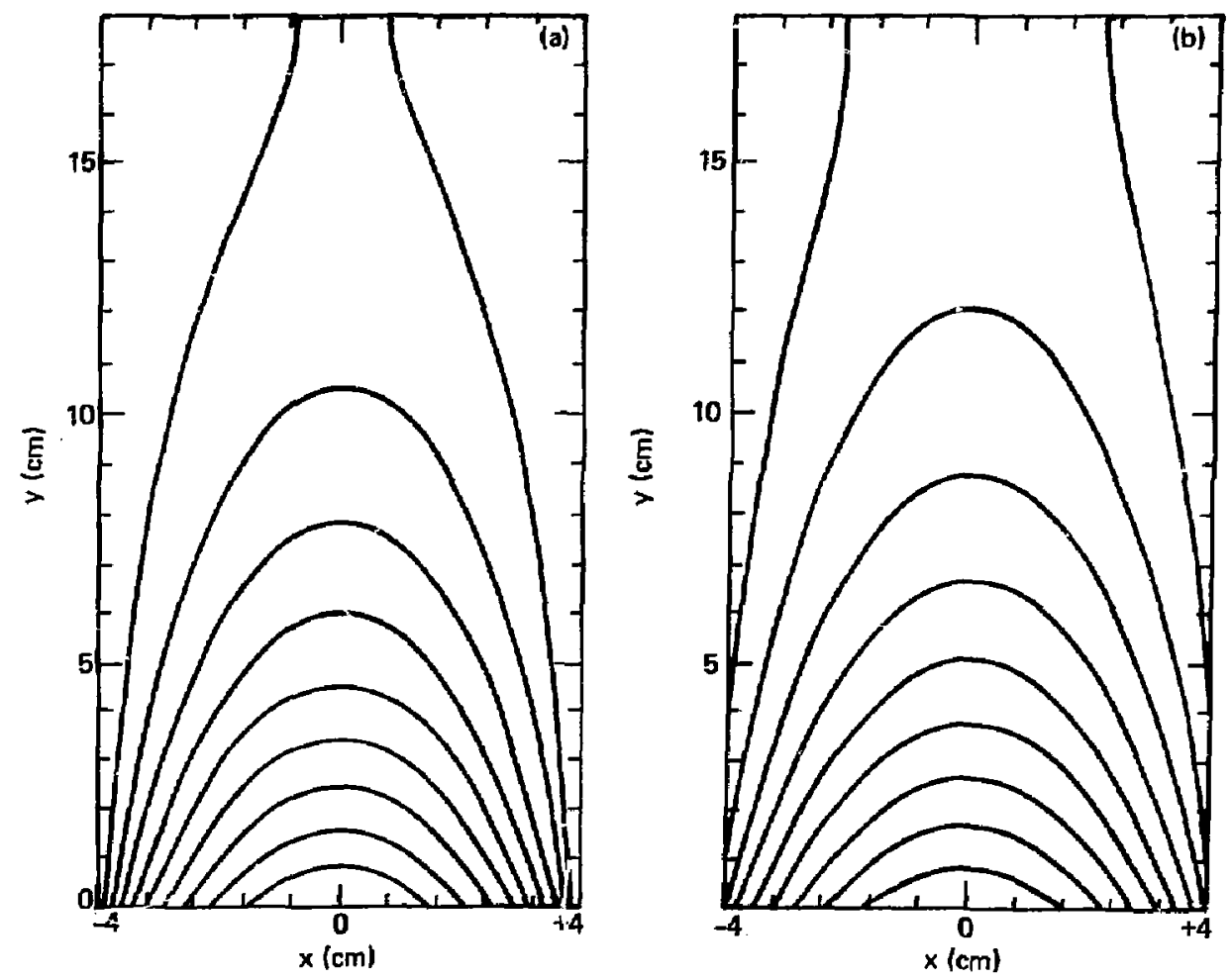

FIG. A3. Cantours of uniform gas density computed for two boundary conditions. The input conditions are those of the third column of Table 1 with $\mathrm{W}=10 \mathrm{~cm}$.

a. GASOUT 1. Uniform density, $n_{G}$ at the poundary of the pumping volumes. The contour interval is $\Delta \mathrm{n}=6.6 \times 10^{13} \mathrm{~cm}^{-3}$.

b. GASOUT 2. Gas density at boundary of pumping volume is determined by gas flow and pumping speed. The contour interval is $\Delta \mathrm{n}=6.7 \times 10^{13} \mathrm{~cm}^{-3}$. 


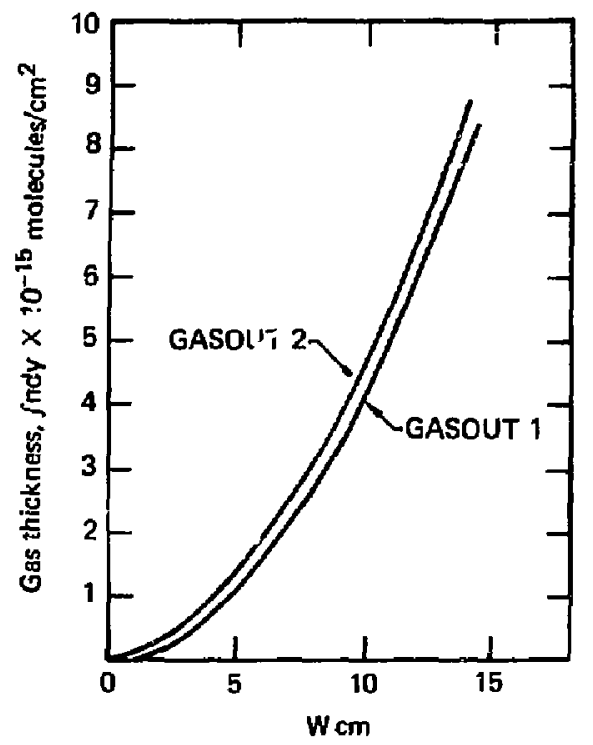

FIli. A4. Integrated gas thickness computed for two boundary conditions with W in the range from 0 to $14 \mathrm{~cm}$. The difference in boundary conditions is important for small values of $\mathrm{H}$. If we can tolerate a gas thickness of $2 \times$ $10^{14} \mathrm{~cm}^{-2} \mathrm{~W}$ must be smaller than about $2 \mathrm{~cm}$. 


\section{REFERENCES}

1. A. W. Maschke, "MEQALAC: A New Approach to Low Beta Acceleration," Brookhayen National Laboratory Report BNL-51029 (1979).

2. C. M. Van Atta, Vacuum Science and Engineering, (McGraw Hi11, New York, 1965), p. 49.

3. H. S. Cars law and J. C. Jaeger, Conduction of Heat in Solids, COxford University Press, Oxford, U.K., 1959).

$E O / s c$ 